\title{
Dynamic Whole-Body Mobile Manipulation with a Torque Controlled Humanoid Robot via Impedance Control Laws
}

\author{
Alexander Dietrich, Thomas Wimböck, and Alin Albu-Schäffer
}

\begin{abstract}
Service robotics is expected to be established in human households and environments within the next decades. Therefore, dexterous and flexible behavior of these systems as well as guaranteeing safe interaction are crucial for that progress. We address these issues in terms of control strategies for the whole body of DLR's humanoid Justin. Via impedance control laws, we enable the robot to realize main tasks compliantly while, at the same time, taking care of aspects like physical limitations and collision avoidance with its own structure and the environment autonomously. The controller provides a natural redundancy resolution between the arms, the torso and the wheeled platform. A low-dimensional task space interface is proposed that can be used by planning tools. Thereby, planning time can be saved significantly. Experimental results on DLR's Justin are presented to validate our approach.
\end{abstract}

\section{INTRODUCTION}

Service and household robots are expected to play an important role in future domestic environments. A high degree of flexibility and the guarantee of a maximum level of safety are two crucial attributes of such robotic systems.

Concerning the first requirement, the ability to move freely in human environment is essential. Beside legged manipulators like Honda's Asimo [1], a wide variety of wheeled mobile robots [2], [3], [4] has been developed to meet that criterion.

The aspect of safety is even more important since injuries and material damage must be avoided in any case. Unpredictable events and the interaction with humans in domestic environments call for sophisticated and adaptive control methods and compliant behavior in particular. Torque sensors in most of Justin's joints (see Fig. 1) enable it to realize such a necessary compliance [5] based torque control and not stiff position control.

Whole-body motion planning and control has been addressed frequently over the last years [6], [7], [8], [9]. In case of wheeled systems, two general alternatives exist in terms of incorporating the mobile base. One separates the dexterous upper body and the platform, and these two subsystems are then controlled independently. However, that requires a synchronization concept [10], [11]. Another approach proposes to control the complete humanoid [8]. In this case the synchronization is not relevant. However, the complexity of the controlled system increases significantly.

As planning of whole-body motions is known to be extremely computing time consuming, it is desirable to

The authors are with the German Aerospace Center (DLR), Institute of Robotics and Mechatronics, Wessling D-82234, Germany; Contact: alexander.dietrich@dlr.de

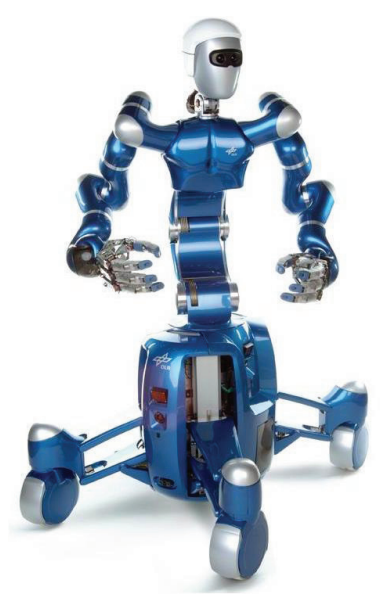

Fig. 1. DLR's humanoid Justin.

reduce the dimensionality [12] instead of accounting for the complete configuration space of the robot. A task like picking up a glass of water and handing it over to the user, for example, would just require the planner to realize a grasping strategy and generate a suitable trajectory for the glass itself. Considering the whole joint space of the robot and issues like collision avoidance, mechanical end stops and an effective synchronization of all body parts would increase the planning time. For these subtasks, reactive control methods based on potential fields [13] are often proposed [14], [10]. In the event of insolvable problems for the local controllers, e.g., local minima, a feedback to the planner can enable to replan the trajectory. In [15], Brock introduced the elastic strips framework approach which allows to execute previously planned motions in a dynamic environment. To this end, the robot reactively adapts to changes in the environment, e.g., when an obstacle is approaching the manipulator.

Another aspect of whole-body motion control concerns the prioritization of competitive tasks. A multitude of established methods is known from literature. In [16], Mansard et al. compare different classical control laws and focuse on discontinuities arising when subtasks get (de-)activated. One possibility to ensure continuity is to apply a damped least square inverse. However, by smoothing the transition, the priority order is not ensured and task execution may be corrupted. In [17], a hierarchy is realized by null space projection techniques and prevents discontinuities concerning unilateral constraints. A whole-body control approach has been proposed in [18], wherein three priority levels are realized which refer to physical constraints, tasks and 
TABLE I

ACTUATED DEGREES OF FREEDOM

\begin{tabular}{|c||c|c|}
\hline Subsystem & DOF & control interface \\
\hline \hline Torso & 3 & torque \\
\hline Arms & $2 \times 7$ & torque \\
\hline Hands & $2 \times 12$ & torque \\
\hline Neck & 2 & position/velocity \\
\hline Platform \& Legs & 8 & position/velocity \\
\hline \hline$\sum$ & 51 & \\
\hline
\end{tabular}

postures. In [10], the so-called dynamical systems approach is used to define transition rates in order to switch smoothly between competitive tasks. A similar solution is presented in [14], wherein different tasks are scaled online, depending on adaptive weighting parameters.

Goal of this paper is to derive a whole-body impedancebased controller for the humanoid Justin to provide a lowdimensional task space interface for higher level planning algorithms. As the underlying control methods are largely known and validated in simulation [18], we focus on the realization on a real robotic system. Therefore, the main contribution is design and experimental evaluation of a compliant behavior for the torque controlled robot containing 51 degrees of freedom. We also provide safe human interaction while tasks are accomplished. By using one single Jacobian for the whole system except for the hands, a synchronized behavior is achieved. We are among the first to implement and analyze such a whole-body motion strategy on a torque controlled humanoid considering a numerousness of different aspects. We assign goal trajectories for the tool center points (TCP) of both arms and not for all joints. Furthermore, we ensure a safe and efficient robot behavior since the high redundancy of the manipulator is utilized to realize subtasks like collision avoidances, different kinds of singularity avoidance, posture primitives, avoidance of mechanical end stops and null space damping. As a consequence of suitable choice and design of these subtasks, it is possible to project all of them into the null space of the main task without inducing undesired competitive behavior. Our experiments demonstrate that, using only two levels of priority and properly designed subtasks within, a multitude of goals can be simultaneously achieved in practice without conflicts.

The paper is organized as follows. After a brief introduction of the employed system in Section II, the control structure is derived in Section III. That comprises joint level control, main task impedance design and redundancy resolution. Thereafter, experimental results on DLR's humanoid Justin are presented and discussed in Section IV.

\section{SYSTEM MODEL}

DLR's humanoid Justin consists of an upper body system which is mounted on a mobile platform, see Fig. 1. The latter is able to realize any translational and rotational trajectory in the plane. Furthermore, the footprint is variable due to four extendable legs. The upper body consists of a torso, two arms, two hands and a sensor head which is mounted on

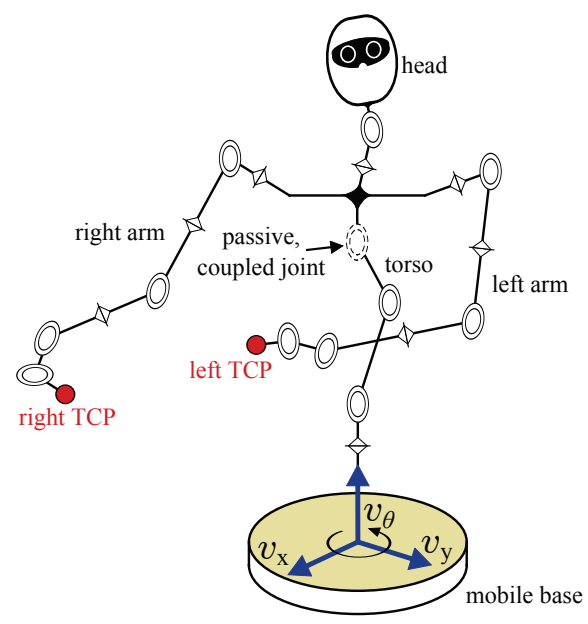

Fig. 2. Kinematic model of the robot illustrating the joints, the TCPs and the moving directions of the mobile base. The platform legs are not depicted.

a pan-tilt unit for unrestricted stereo vision in the working range of the manipulator. Except for the two neck joints, each actuated upper body joint has a built-in torque sensor and allows torque control accordingly. Fig. 2 depicts the kinematic structure of the robot except for the hands and the extendable legs. The possible moving directions of the platform are represented by the velocities $v_{\mathrm{x}}, v_{\mathrm{y}}$ and $v_{\theta}$. The total number of 51 actuated degrees of freedom (DOF) is grouped by subsystem and control interface in Table I.

The upper body weighs approximately $45 \mathrm{~kg}$, whereas the mobile platform amounts to about $150 \mathrm{~kg}$. Further details on the structure, kinematics and control can be found in [19], [20].

The equations of motion for the system can be expressed by

$$
\begin{aligned}
& {\left[\begin{array}{ll}
\boldsymbol{M}_{\mathrm{ww}} & \boldsymbol{M}_{\mathrm{wq}} \\
\boldsymbol{M}_{\mathrm{wq}}^{T} & \boldsymbol{M}_{\mathrm{qq}}
\end{array}\right]\left(\begin{array}{c}
\ddot{\boldsymbol{w}} \\
\ddot{\boldsymbol{q}}
\end{array}\right)+\boldsymbol{C}(\boldsymbol{w}, \dot{\boldsymbol{w}}, \boldsymbol{q}, \dot{\boldsymbol{q}})+} \\
& +\left(\begin{array}{c}
\boldsymbol{g}_{\mathrm{b}}(\boldsymbol{w}) \\
\boldsymbol{g}_{\mathrm{q}}(\boldsymbol{q})
\end{array}\right)=\left(\begin{array}{c}
\boldsymbol{\tau}_{\mathrm{w}} \\
\boldsymbol{\tau}_{\mathrm{q}}
\end{array}\right)+\boldsymbol{\tau}_{\text {ext }} .
\end{aligned}
$$

The vector $\boldsymbol{q} \in \mathbb{R}^{43}$ denotes the upper body joint coordinates and $\boldsymbol{w} \in \mathbb{R}^{8}$ comprises the steering and spinning angles of the four wheels. On the left hand side the variable inertia matrix is shown. Centripetal/Coriolis effects are accounted for by the term $\boldsymbol{C}(\boldsymbol{w}, \dot{\boldsymbol{w}}, \boldsymbol{q}, \dot{\boldsymbol{q}})$. The gravity torque that appears at the joints of the upper body is represented by $\boldsymbol{g}_{\mathrm{q}}(\boldsymbol{q})$. Analogously, $\boldsymbol{g}_{\mathrm{b}}(\boldsymbol{w})$ is defined for the base. External torques and forces are expressed by $\tau_{\text {ext }}$. Control inputs are $\tau_{\mathrm{w}}$ and $\tau_{\mathrm{q}}$. Other physical effects like link and joint flexibility as well as joint friction are neglected in the representation of (1). Actually, some of these effects are incorporated and compensated by subordinate control loops as described in the next section.

\section{CONTROL ALGORITHM}

In this section, the control algorithm for whole-body motions is derived. Starting with a short overview of the 
proposed structure of the dynamic controller, we proceed with the subordinate joint level controllers. Having the basic architecture, we formulate the mechanical impedance laws [21] to realize the main task. Then, we provide a redundancy resolution to account for subtasks and physical constraints.

\section{A. Dynamic Controller}

1) Control Algorithm Overview: The schematic wholebody motion concept is visualized in Fig. 3. Fundamentally, one can divide the structure into three separate parts.

In the center, the main task realization is depicted. After suitable trajectory generation, the impedance law is applied and leads to desired joint torques/forces. The mobile base is controlled kinematically. Hence, we employ an admittance interface. On the right, the robot model is shown. The torque controlled upper body and the velocity controlled mobile base provide measurements $\boldsymbol{q}$ and an estimation of the platform odometry $\boldsymbol{X}_{\text {odo. }}$. On the left, security-relevant collision avoidances are realized and added to the main task commands. A separate low level priority block contains several subtasks which are projected into the null space of the main task.

2) Joint Level Control: Based on the general model (1), we make some assumptions for further analyses:

Assumption 1: A kinematic-based platform motion controller [20] is able to compensate any dynamic effects and realizes any given velocity profile within the feasible range of acceleration and velocity.

Concerning (1), the assumption means that any dynamic effects in the first row are negligible. The motion controller which is based on dynamic feedback linearization enables to track a given velocity profile in the three moving directions indicated by $\boldsymbol{v}_{\mathrm{x}}, \boldsymbol{v}_{\mathrm{y}}$ and $\boldsymbol{v}_{\theta}$ in Fig. 2.

Assumption 2: Centripetal/Coriolis effects and a mass coupling between upper body and the mobile base can be neglected.

For the controller design, we separate the upper body from the platform. Therefore, the inertia element $M_{\text {wq }}$ from (1) is omitted. We also leave out $\boldsymbol{C}(\boldsymbol{w}, \dot{\boldsymbol{w}}, \boldsymbol{q}, \dot{\boldsymbol{q}})$ as our controller is not supposed to be a tracking controller. It shall be noted that accounting for centrifugal/Coriolis effects can be realized via a feed-forward term as shown in [22].

Assumption 3: Ideal torque sources allow to realize any given $\tau_{\mathrm{q}}$.

This assumption holds because $\tau_{\mathrm{q}}$ is realized by a torque feedback [23] which incorporates the joint torque, the motor position and their derivatives. Friction is also compensated.

In order to apply a desired force/torque ${ }^{1} \tau_{\mathrm{b}} \in \mathbb{R}^{3}$ to the platform with respect to the three directions, an admittance interface is proposed:

$$
\boldsymbol{M}_{\mathrm{b}} \dot{\boldsymbol{v}}^{\mathrm{d}}+\boldsymbol{D}_{\mathrm{b}} \boldsymbol{v}^{\mathrm{d}}=\boldsymbol{\tau}_{\mathrm{b}} .
$$

The commanded velocities are expressed by $\boldsymbol{v}^{\mathrm{d}} \in \mathbb{R}^{3}$. A desired virtual platform inertia can be realized by $M_{\mathrm{b}}$.

\footnotetext{
${ }^{1}$ Depending on the type of joint (revolute or prismatic), the respective element is of dimension $[\mathrm{Nm}]$ or $[\mathrm{N}]$, respectively.
}

Analogously, damping is injected via $\boldsymbol{D}_{\mathrm{b}}$. More natural motions of the platform can be achieved by adding the upper body inertial coupling to the left hand side in (2).

Summarized, one can say that through $\tau_{\mathrm{q}}$ from (1) and $\tau_{\mathrm{b}}$ from (2), any upper body configuration can be reached as well as the tracking of arbitrary translational and rotational platform trajectories.

3) Impedance Design: Using $\tau_{\mathrm{q}}$ from (1) and $\tau_{\mathrm{b}}$ from (2), one has access to all necessary joint motions. Now the question arises how to set the control inputs. In this approach, we use a superposition of variously weighted tasks. In the following we derive the required values for the main task, that is an impedance behavior to follow the planned TCP trajectory.

One well-known impedance design is given by the following equation and leads to the upper body joint torques $\tau:$

$$
\boldsymbol{\tau}=\boldsymbol{g}_{\mathrm{q}}(\boldsymbol{q})-\left(\frac{\partial V_{\mathrm{imp}}\left(\boldsymbol{q}, \boldsymbol{X}_{\mathrm{odo}}\right)}{\partial \boldsymbol{q}}\right)^{T}-\boldsymbol{D}_{\mathrm{imp}}(\boldsymbol{q}) \dot{\boldsymbol{q}}
$$

Gravity compensation is realized by $\boldsymbol{g}_{\mathrm{q}}(\boldsymbol{q})$ and a spatial spring by the potential $V_{\mathrm{imp}}\left(\boldsymbol{q}, \boldsymbol{X}_{\text {odo }}\right)$. Additional damping is injected through $\boldsymbol{D}_{\text {imp }}(\boldsymbol{q})$. From a passivity point of view, any positive semi-definite matrix can be applied. Within this framework, $\boldsymbol{D}_{\mathrm{imp}}(\boldsymbol{q})$ is chosen such that desired damping ratios are realized. This can be achieved, for example, by a double diagonalization $^{2}$ of the inertia matrix and the Hessian of the potential function, i.e., $\partial^{2} V_{\text {imp }}\left(\boldsymbol{q}, \boldsymbol{X}_{\text {odo }}\right) / \partial \boldsymbol{q}^{2}$.

The potential according to a spatial spring which is spanned between two frames ${ }^{3} \boldsymbol{H}_{1} \in S E(3)$ and $\boldsymbol{H}_{2} \in$ $S E(3)$ will be denoted by $V_{\mathrm{s}}\left(\boldsymbol{H}_{1}, \boldsymbol{H}_{2}, \mathcal{K}\right)$ following the notation in [25]. The spatial stiffness is parameterized by $\mathcal{K}$. Correspondingly, $V_{\text {imp }}\left(\boldsymbol{q}, \boldsymbol{X}_{\text {odo }}\right)$ can be expressed by

$$
\begin{aligned}
V_{\mathrm{imp}}\left(\boldsymbol{q}, \boldsymbol{X}_{\text {odo }}\right)= & V_{\mathrm{s}}\left(\boldsymbol{H}_{\mathrm{r}}\left(\boldsymbol{q}, \boldsymbol{X}_{\text {odo }}\right), \boldsymbol{H}_{\mathrm{r}}^{\mathrm{d}}, \mathcal{K}_{\mathrm{r}}\right)+ \\
& V_{\mathrm{s}}\left(\boldsymbol{H}_{\mathrm{l}}\left(\boldsymbol{q}, \boldsymbol{X}_{\text {odo }}\right), \boldsymbol{H}_{\mathrm{l}}^{\mathrm{d}}, \mathcal{K}_{\mathrm{l}}\right)
\end{aligned}
$$

since both the right and the left TCP are regarded (subscripts $\mathrm{r}$ and 1). The respective desired equilibrium frames are indicated by superscript $\mathrm{d}$.

Within this work, we require the Jacobian matrices for the TCPs. Thus, we split up into Cartesian force/torque calculation and projection into joint space. Extraction of local Cartesian coordinates $\boldsymbol{x}_{\mathrm{r}}\left(\boldsymbol{q}, \boldsymbol{X}_{\text {odo }}\right), \boldsymbol{x}_{\mathrm{r}}^{\mathrm{d}}, \boldsymbol{x}_{1}\left(\boldsymbol{q}, \boldsymbol{X}_{\text {odo }}\right), \boldsymbol{x}_{\mathrm{l}}^{\mathrm{d}} \in$ $\mathbb{R}^{6 \times 1}$ from the respective frames in (4) and applying a Cartesian stiffness $\boldsymbol{K}_{\mathrm{c}}$ leads to the forces

$$
\left(\begin{array}{l}
\boldsymbol{F}_{\mathrm{r}, \mathrm{k}} \\
\boldsymbol{F}_{\mathrm{l}, \mathrm{k}}
\end{array}\right)=-\boldsymbol{K}_{\mathrm{c}} \cdot\left(\begin{array}{l}
\boldsymbol{x}_{\mathrm{r}}\left(\boldsymbol{q}, \boldsymbol{X}_{\text {odo }}\right)-\boldsymbol{x}_{\mathrm{r}}^{\mathrm{d}} \\
\boldsymbol{x}_{\mathrm{l}}\left(\boldsymbol{q}, \boldsymbol{X}_{\text {odo }}\right)-\boldsymbol{x}_{\mathrm{l}}^{\mathrm{d}}
\end{array}\right) .
$$

\footnotetext{
${ }^{2}$ Given a symmetric and positive definite matrix $\boldsymbol{A} \in \mathbb{R}^{n \times n}$ and a symmetric matrix $\boldsymbol{B} \in \mathbb{R}^{n \times n}$, a nonsingular matrix $\boldsymbol{Q} \in \mathbb{R}^{n \times n}$ and a diagonal matrix $\boldsymbol{B}_{0} \in \mathbb{R}^{n \times n}$ can be found such that $\boldsymbol{Q}^{T} \boldsymbol{Q}=\boldsymbol{A}$ and $\boldsymbol{B}=\boldsymbol{Q}^{T} \boldsymbol{B}_{0} \boldsymbol{Q}[24]$.

${ }^{3}$ Herein, $S E(3)$ denotes the special Euclidean group and element $\boldsymbol{H} \in$ $S E(3)$ can be written as $\boldsymbol{H}=[\boldsymbol{R}, \boldsymbol{p}]$, wherein $\boldsymbol{R} \in S O(3)$ expresses a rotation matrix and $\boldsymbol{p} \in \mathbb{R}^{3}$ a translation vector.
} 


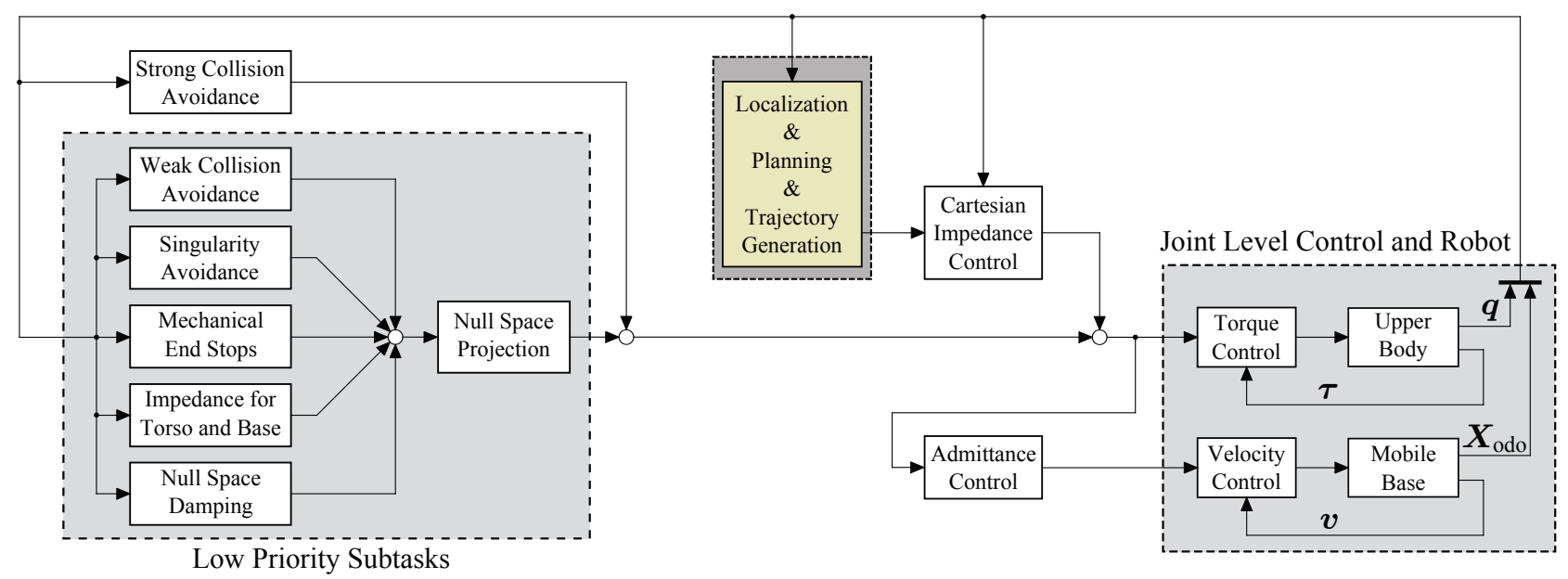

Fig. 3. Control architecture for whole-body motion.

Additionally, a Cartesian damping can be injected w.r.t. the Cartesian TCP velocities:

$$
\left(\begin{array}{c}
\boldsymbol{F}_{\mathrm{r}, \mathrm{d}} \\
\boldsymbol{F}_{\mathrm{l}, \mathrm{d}}
\end{array}\right)=-\boldsymbol{D}_{\mathrm{c}} \cdot\left(\begin{array}{c}
\dot{\boldsymbol{x}}_{\mathrm{r}}(\boldsymbol{q}, \dot{\boldsymbol{q}}) \\
\dot{\boldsymbol{x}}_{1}(\boldsymbol{q}, \dot{\boldsymbol{q}})
\end{array}\right) .
$$

The damping matrix $\boldsymbol{D}_{\mathrm{c}} \in \mathbb{R}^{12 \times 12}$ can be computed as described above using $\boldsymbol{K}_{\mathrm{c}}$ from (5) and the projected TCP inertia matrices. For the latter projection the Jacobian $\boldsymbol{J}$ is required which is composed of submatrices corresponding to the participating body links in the kinematic chain:

$$
\boldsymbol{J}(\boldsymbol{q})=\left[\begin{array}{llcc}
\boldsymbol{J}_{\mathrm{r}, \text { base }} & \boldsymbol{J}_{\mathrm{r}, \text { torso }} & \boldsymbol{J}_{\mathrm{r}, \text { arm }} & \mathbf{0} \\
\boldsymbol{J}_{1, \text { base }} & \boldsymbol{J}_{1, \text { torso }} & \mathbf{0} & \boldsymbol{J}_{1, \text { arm }}
\end{array}\right] .
$$

To obtain the required joint torques/forces for realizing the chosen impedance, the necessary Cartesian commands from (5) and (6) have to be projected into joint space using $\boldsymbol{J}(\boldsymbol{q})$. At this point also gravity compensation has to be considered.

$$
\left(\begin{array}{c}
\boldsymbol{\tau}_{\mathrm{b}, \text { cart }} \\
\boldsymbol{\tau}_{\mathrm{q}, \text { cart }}
\end{array}\right)=\boldsymbol{J}(\boldsymbol{q})^{T} \cdot\left(\begin{array}{l}
\boldsymbol{F}_{\mathrm{r}, \mathrm{k}}+\boldsymbol{F}_{\mathrm{r}, \mathrm{d}} \\
\boldsymbol{F}_{1, \mathrm{k}}+\boldsymbol{F}_{\mathrm{l}, \mathrm{d}}
\end{array}\right)+\left(\begin{array}{c}
\mathbf{0} \\
\boldsymbol{g}_{\mathrm{q}}(\boldsymbol{q})
\end{array}\right) .
$$

Herein, $\tau_{\mathrm{b}, \text { cart }}$ denotes the joint torques/forces which result for the mobile base and $\tau_{\mathrm{q} \text {,cart }}$ represents the joint torques for the whole upper body without the head.

The hands can also be included in the Jacobian to realize the impedance. However, in this framework we separate macro-manipulation (guiding the TCPs to the desired Cartesian positions) and micro-manipulation (dexterous manipulation using the hands).

\section{B. Redundancy Resolution}

Since the whole-body motion is to be coordinated by the controller itself and not by a planner, different issues have to be tackled simultaneously. These are:

1) Upper Body Self-Collision Avoidance: A potential field-based approach for avoiding collisions with the structure is proposed in [26]. The algorithm accounts for a variable number of potentially colliding body links simultaneously. Virtual collision points on tight convex hulls around the robot structure are calculated in real-time. Subsequently, repulsive forces are applied there and projected into the joint space:

$$
\boldsymbol{\tau}_{\mathrm{q}, \mathrm{ca}}=-\left(\frac{\partial V_{\mathrm{q}, \mathrm{ca}}(\boldsymbol{q})}{\partial \boldsymbol{q}}\right)^{T}-\boldsymbol{D}_{\mathrm{q}, \mathrm{ca}}(\boldsymbol{q}) \dot{\boldsymbol{q}}
$$

This passivity-based algorithm comprises a configuration dependent damping which is injected via $\boldsymbol{D}_{\mathrm{q}, \mathrm{ca}}(\boldsymbol{q})$. Design parameters concerning the repulsion potential $V_{\mathrm{q}, \mathrm{ca}}(\boldsymbol{q})$ are the respective potential stiffness and spatial extension.

2) Platform Collision Avoidance: Since the platform is kinematically controlled, a collision with obstacles would not be recognized by any force or torque sensors. Therefore, time-of-flight cameras have been mounted on each of the four sides of the mobile base, see Fig. 1. Using this data enables to generate a repulsive potential field $V_{\mathrm{b}, \mathrm{ca}}$ around the mobile platform, analog to (9):

$$
\boldsymbol{\tau}_{\mathrm{b}, \mathrm{ca}}=-\left(\frac{\partial V_{\mathrm{b}, \mathrm{ca}}\left(\boldsymbol{X}_{\mathrm{odo}}, \boldsymbol{X}_{\mathrm{obs}}\right)}{\partial \boldsymbol{X}_{\mathrm{odo}}}\right)^{T} .
$$

Potential obstacles are accounted for by $\boldsymbol{X}_{\text {obs }}$. A damping term can be omitted here since energy dissipation is already realized by $\boldsymbol{D}_{\mathrm{b}}$ in the admittance law (2).

3) Static Singularity Avoidance for the Arms: To avoid singular configurations of the two arms and hence rank deficiency of the respective Jacobian, a potential can be generated which is based on the manipulability measure:

$$
m_{\text {kin }}(\boldsymbol{q})=\sqrt{\operatorname{det}\left(\boldsymbol{J}_{\mathrm{arms}}(\boldsymbol{q}) \boldsymbol{J}_{\mathrm{arms}}^{T}(\boldsymbol{q})\right)}
$$

where $\boldsymbol{J}_{\text {arms }}(\boldsymbol{q})$ denotes a submatrix of (7) which contains the left and the right arm Jacobian. The respective potential field is defined as

$$
V_{\mathrm{q}, \mathrm{sa}}(\boldsymbol{q})=\left\{\begin{array}{ll}
k_{\mathrm{s}}\left(m_{\mathrm{kin}}(\boldsymbol{q})-m_{0}\right)^{2} & m_{\mathrm{kin}}(\boldsymbol{q}) \leq m_{0} \\
0 & m_{\mathrm{kin}}(\boldsymbol{q})>m_{0}
\end{array},\right.
$$

with $k_{\mathrm{S}}$ describing a positive scalar factor to control the gain of the singularity avoidance. An upper bound $m_{0}>0 \in \mathbb{R}$ 
for $m_{\text {kin }}(\boldsymbol{q})$ determines the transition between active and inactive singularity avoidance control [27]. The required avoidance torque vector can be obtained from (13).

$$
\boldsymbol{\tau}_{\mathrm{q}, \mathrm{sa}}=-\left(\frac{\partial V_{\mathrm{q}, \mathrm{sa}}(\boldsymbol{q})}{\partial \boldsymbol{q}}\right)^{T}
$$

It shall be mentioned that a manipulability measure w.r.t. the entire Jacobian $\boldsymbol{J}(\boldsymbol{q})$ is also possible. However, due to the numerousness of DOF, its evaluation would be expensive in terms of computing time.

4) Dynamic Singularity Avoidance for the Platform: Depending on the location of the instantaneous center of rotation further singularities may arise concerning the mobile base kinematics. Allowing those undesirable dynamic configurations would result in unfeasible wheel steering rates and generate high internal forces which stress the mechanical structure. A reactive potential field-based controller is proposed in [28] which outputs avoidance accelerations

$$
\dot{\boldsymbol{v}}_{\mathrm{b}, \mathrm{sa}}=-\left(\frac{\partial V_{\mathrm{b}, \mathrm{sa}}(\boldsymbol{z})}{\partial \boldsymbol{v}}\right)^{T}=\left(\begin{array}{c}
\dot{v}_{\mathrm{x}, \mathrm{sa}} \\
\dot{v}_{\mathrm{y}, \mathrm{sa}} \\
\dot{v}_{\theta, \mathrm{sa}}
\end{array}\right)
$$

with $V_{\mathrm{b}, \mathrm{sa}}(\boldsymbol{z})$ as the respective potential and the location $\boldsymbol{z}$ of the instantaneous center of rotation defined as a function of the platform DOF in body coordinates or the base velocity $\boldsymbol{v}$, respectively:

$$
z=\mathcal{F}(\boldsymbol{w}, \dot{\boldsymbol{w}}, \boldsymbol{\lambda})=\mathcal{G}(\boldsymbol{v})
$$

The leg lengths are expressed by $\boldsymbol{\lambda}$. In order to apply the translational and rotational accelerations $\dot{v}_{\mathrm{x}, \mathrm{sa}}, \dot{v}_{\mathrm{y} \text {,sa }}$ and $\dot{v}_{\theta \text {,sa }}$ using the proposed admittance (2), a transformation into applicable forces/torques $\tau_{\mathrm{b}, \mathrm{sa}}$ has to be performed:

$$
\tau_{\mathrm{b}, \mathrm{sa}}=M_{\mathrm{b}} \dot{\boldsymbol{v}}_{\mathrm{b}, \mathrm{sa}} .
$$

The virtual mass $M_{\mathrm{b}}$ from (2) has been used here to ensure that the accelerations are applied unmodified when $\tau_{\mathrm{b}, \mathrm{sa}}$ or its null space projection, respectively, enters the platform admittance (2).

5) Mechanical End Stops: In order to sustain manipulability, it is also necessary to avoid physical joint limits $\boldsymbol{q}_{\text {stop }}$. To achieve that, unilateral virtual springs have been designed around the end stops.

$$
\boldsymbol{\tau}_{\mathrm{q}, \lim }=-\left(\frac{\partial V_{\mathrm{q}, \lim }\left(\boldsymbol{q}, \boldsymbol{q}_{\mathrm{stop}}\right)}{\partial \boldsymbol{q}}\right)^{T}
$$

wherein $V_{\mathrm{q}, \mathrm{lim}}\left(\boldsymbol{q}, \boldsymbol{q}_{\mathrm{stop}}\right)$ denotes the respective potential.

6) Desired Impedance for Torso and Mobile Base: For providing an unrestricted stereo vision, specific configurations of the torso support the neck joint actuators in positioning the camera system which is mounted on the head. Likewise, a separate trajectory for the mobile base allows to handle obstacles in the workspace of the robot which are known in advance. Otherwise, the latter can only be achieved by affecting the platform indirectly through TCP trajectories which is not very effective. A potential function can be set up including these two issues (subscript $t$ for torso and $b$ for mobile base):

$$
\begin{aligned}
V_{\mathrm{tb}, \operatorname{imp}}\left(\boldsymbol{q}, \boldsymbol{X}_{\text {odo }}\right)= & V_{\mathrm{s}}\left(\boldsymbol{H}_{\mathrm{t}}(\boldsymbol{q}), \boldsymbol{H}_{\mathrm{t}}^{\mathrm{d}}, \mathcal{K}_{\mathrm{t}}\right)+ \\
& V_{\mathrm{s}}\left(\boldsymbol{H}_{\mathrm{b}}\left(\boldsymbol{X}_{\text {odo }}\right), \boldsymbol{H}_{\mathrm{b}}^{\mathrm{d}}, \mathcal{K}_{\mathrm{b}}\right) .
\end{aligned}
$$

For determining the current frame of the mobile base $\boldsymbol{H}_{\mathrm{b}}$, the odometry $\boldsymbol{X}_{\text {odo }}$ is required here. Consequently, the required inputs can be computed as follows:

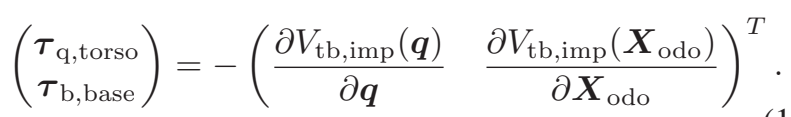

7) Null Space Damping: A damping in joint space is able to ensure that no undesirable null space oscillations build up.

$$
\boldsymbol{\tau}_{\mathrm{q}, \mathrm{d}}=-\boldsymbol{D}_{\mathrm{q}, \mathrm{d}}(\boldsymbol{q}) \dot{\boldsymbol{q}} .
$$

Herein, $\boldsymbol{D}_{\mathrm{q}, \mathrm{d}}(\boldsymbol{q})$ describes an arbitrary positive definite damping matrix and $\tau_{\mathrm{q}, \mathrm{d}}$ represents the respective torques.

The question arises how to take into account the large number of different tasks/subtasks. A prioritization has to be performed since it is impossible to realize all tasks simultaneously and equally in particular. As a high degree of safety is one of the key features, collision avoidance has the top priority. Also, the precise execution of the main task is settled at the top. Nevertheless, having two tasks at the same priority level can cause competitive behavior.

Since safety aspects are even more important than task execution, both upper body and platform collision avoidance are to be designed twice. In the first case, tight and very strong repulsion fields are set to the highest priority, together with task execution (see Fig. 3). The second case which includes weaker and largely extended fields is used on a lower priority level. That utilizes the redundancy to keep the robot always in a "good" configuration. Task execution is not disturbed except for limit configurations and cases. Then, the strong collision avoidance repulsion gets activated and ensures safety. Therefore, top priority comprises task execution $\left(\tau_{\mathrm{b}, \text { cart }}, \tau_{\mathrm{q}, \text { cart }}\right)$ and tight/strong collision avoidance, denoted by $\boldsymbol{\tau}_{\mathrm{b}, \mathrm{ca}, \mathrm{I}}$ and $\boldsymbol{\tau}_{\mathrm{q}, \mathrm{ca}, \mathrm{I}}$.

On lower priority level, weaker collision avoidances $\left(\boldsymbol{\tau}_{\mathrm{b}, \mathrm{ca}, \mathrm{II}}, \boldsymbol{\tau}_{\mathrm{q}, \mathrm{ca}, \mathrm{II}}\right.$ without damping, i.e., $\boldsymbol{D}_{\mathrm{q}, \mathrm{ca}, \mathrm{II}}(\boldsymbol{q})=\mathbf{0}$ ) as well as separate damping $\tau_{\mathrm{q}, \mathrm{d}}$, singularity avoidances $\left(\tau_{\mathrm{b}, \mathrm{sa}}, \tau_{\mathrm{q}, \mathrm{sa}}\right)$, additional impedances for torso and mobile base $\left(\boldsymbol{\tau}_{\mathrm{q}, \text { torso }}, \boldsymbol{\tau}_{\mathrm{b} \text {,base }}\right)$ and the incorporation of mechanical end stops $\tau_{\mathrm{q}, \mathrm{lim}}$ remain.

Although a numerousness of tasks are at the same priority level, no critical competitive behavior is expected:

- Weak collision avoidances $\boldsymbol{\tau}_{\mathrm{b}, \mathrm{ca}, \mathrm{II}}$ and $\boldsymbol{\tau}_{\mathrm{q}, \mathrm{ca}, \mathrm{II}}$ are intended to be outplayed by the other tasks if necessary since $\boldsymbol{\tau}_{\mathrm{b}, \mathrm{ca}, \mathrm{I}}$ and $\boldsymbol{\tau}_{\mathrm{q}, \mathrm{ca}, \mathrm{I}}$ remain at top priority level and guarantee safety in any case.

- Singularity avoidances $\boldsymbol{\tau}_{\mathrm{b}, \mathrm{sa}}$ and $\boldsymbol{\tau}_{\mathrm{q}, \mathrm{sa}}$ are related to different subsystems and have no intersection. 
- The virtual springs with respect to the upper body mechanical end stops and the static singularity avoidance have an intersection. However, singular configurations and mechanical end stops of the arms are not supposed to compete in an undesired manner. Firstly, the tight potential fields of the end stops are designed progressively when approaching the limits so as to be able to absorb much kinetic energy. The singularity avoidance, on the other side, has a wider working range but does not comprise such a high-energy potential as maintaining the manipulability is less important than protecting the mechanics. Secondly, an end stop potential refers to one single joint and the singularity avoidance to all arm joints. In general, there is a way out of a singularity and off one mechanical end stop simultaneously.

- The additional impedances for torso and mobile base do not compete as they refer to different subsystems. The same holds for the arm singularity avoidance. Since the torso is to move in a feasible range only, no interference between $\tau_{\mathrm{q} \text {,torso }}$ and $\tau_{\mathrm{q}, \mathrm{lim}}$ will occur. A competition between $\tau_{\mathrm{b}, \mathrm{sa}}$ and $\tau_{\mathrm{b} \text {,base }}$ may happen. However, the platform singularity avoidance is designed such that it deviates a desired platform motion systematically as described in [28].

- Null space damping $\tau_{\mathrm{q}, \mathrm{d}}$ dissipates kinetic energy from the upper body null space dynamics and thus, it has no problematic influence on the other upper body subtasks on same priority level.

Due to the design of the subtasks, local minima can be largely avoided. Nevertheless, it shall be noted that our redundancy resolution technique is not generic but optimized for our specific system.

We restrict the lower level prioritized subtasks to the null space of the main task, i.e.,

$$
\left(\begin{array}{c}
\boldsymbol{\tau}_{\mathrm{b}} \\
\boldsymbol{\tau}_{\mathrm{q}}
\end{array}\right)=\left(\begin{array}{l}
\boldsymbol{\tau}_{\mathrm{b}, \mathrm{cart}} \\
\boldsymbol{\tau}_{\mathrm{q}, \mathrm{cart}}
\end{array}\right)+\left(\begin{array}{c}
\boldsymbol{\tau}_{\mathrm{b}, \mathrm{ca}, \mathrm{I}} \\
\boldsymbol{\tau}_{\mathrm{q}, \mathrm{ca}, \mathrm{I}}
\end{array}\right)+\mathcal{N}(\boldsymbol{J}) \cdot\left(\begin{array}{l}
\boldsymbol{\tau}_{\mathrm{b}, \text { null }} \\
\boldsymbol{\tau}_{\mathrm{q}, \text { null }}
\end{array}\right)
$$

with the null space projection matrix $\mathcal{N}(\boldsymbol{J})$ and the null space torques/forces represented by the superposition

$$
\begin{aligned}
& \tau_{\mathrm{b}, \text { null }}=\tau_{\mathrm{b}, \mathrm{ca}, \mathrm{II}}+\tau_{\mathrm{b}, \mathrm{sa}}+\tau_{\mathrm{b}, \mathrm{base}}, \\
& \tau_{\mathrm{q}, \text { null }}=\tau_{\mathrm{q}, \mathrm{ca}, \mathrm{II}}+\tau_{\mathrm{q}, \mathrm{sa}}+\tau_{\mathrm{q}, \mathrm{lim}}+\tau_{\mathrm{q}, \text { torso }}+\tau_{\mathrm{q}, \mathrm{d}} .
\end{aligned}
$$

The null space projection matrix from (21) is defined as

$$
\mathcal{N}(\boldsymbol{J})=\boldsymbol{I}-\boldsymbol{J}^{T}\left(\boldsymbol{J}^{+}\right)^{T}
$$

with $I$ expressing the identity matrix. Since the derivation of the generalized inverse $\boldsymbol{J}^{+}$is not unique, a constraint has to be introduced, namely the weighting matrix $\boldsymbol{A}$ :

$$
\boldsymbol{J}^{+\boldsymbol{A}}=\boldsymbol{A} \boldsymbol{J}^{T}\left(\boldsymbol{J} \boldsymbol{A} \boldsymbol{J}^{T}\right)^{-1} .
$$

By substituting $\boldsymbol{A}$ by the inverse of the inertia matrix, a dynamically consistent projection is provided [12].

Applying (21) to the joint level controllers imposed in Sec. III-A.2 completes the control law.

\section{RESULTS}

In the following, three experiments are conducted to demonstrate the performance of the proposed control structure. First, the step response of the right TCP in the case of a forward motion $(0.2 \mathrm{~m})$ is evaluated. As it can be seen in Fig. 4, the actual time constant is about $0.28 \mathrm{~s}$. Besides, the overshooting is mentionable which can be traced back to the delayed behavior of the platform due to the admittance coupling, see Fig. 4 (bottom), and a damping ratio of 0.7 (underdamped) in the impedance. As the impedance is basically a PD-controller without an integrating component, a steady-state error may remain which can also be seen in the figure. An excitation in $x$-direction affects the other two translational directions marginally. The steady-state errors can be reduced by using a higher translational stiffness which is set to $500 \mathrm{~N} / \mathrm{m}$ in this experiment.

The second experiment shows the robot behavior for a continuous trajectory (see first chart in Fig. 6). The initial configuration is depicted in Fig. 5 (left). The right TCP frame is commanded to move forward $1 \mathrm{~m}$, see Fig. 5 (center), and then back to the initial frame, see Fig. 5 (right). Apparently, the control leads to a totally different configuration when approaching the initial frame again. The second diagram in Fig. 4 depicts a norm ${ }^{4}$ of selected null space subtasks to allow easy comparison. Obviously, returning does not lead to the same subtask participation. For example, the upper body singularity avoidance is more crucial while moving forward to prevent the outstreched arm than it is when moving backwards. The third diagram depicts the norms of each addend from (21). The last chart illustrates the base velocities which are output of the admittance (2). The significantly different configuration in Fig. 5 (right) in comparison to the initial pose (left) is primarily caused by the asymmetrical

\footnotetext{
${ }^{4}$ The respective norm of $\boldsymbol{\tau}_{\text {task }}$ is $\frac{10^{-3}}{(\mathrm{Nm})^{2}} \boldsymbol{\tau}_{\text {task }}^{T} \boldsymbol{\tau}_{\text {task }}$. Forces in $\boldsymbol{\tau}_{\text {task }}$ are multiplied with $1 \mathrm{~m}$ beforehand.
}
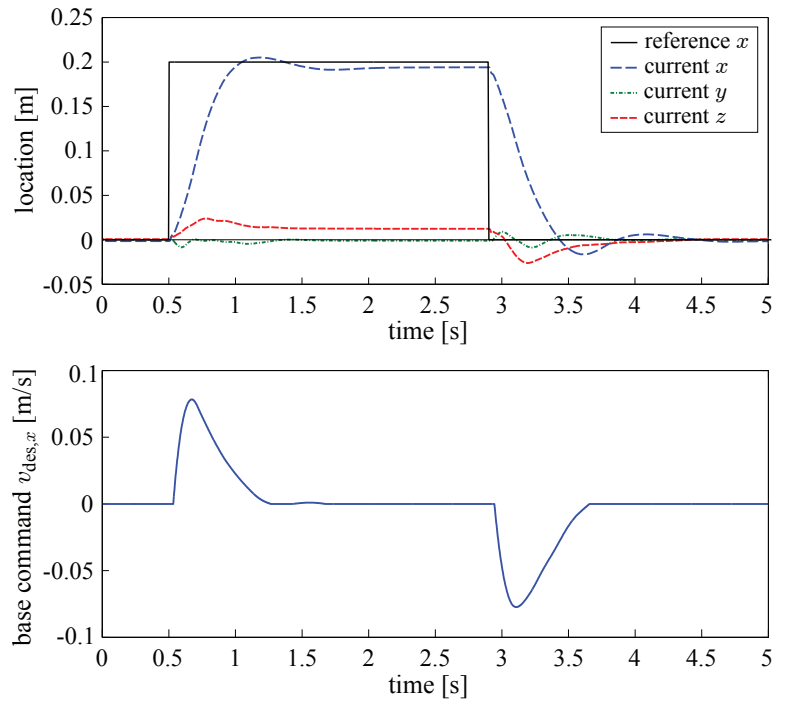

Fig. 4. Step response for a translational TCP motion in $x$-direction with an impedance stiffness of $500 \mathrm{~N} / \mathrm{m}$. 

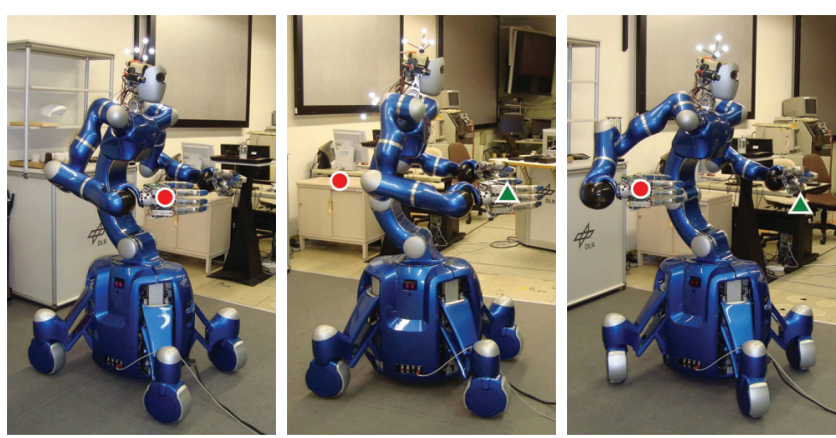

Fig. 5. Snapshots during the experiment. (red circle: initial location of right TCP; green triangle: intermediate location of right TCP)

commands for the mobile platform.

The third experiment shows the performance of the controller while a human is interacting with the robot, see Fig. 7. The user pushes the right TCP away from its desired position and orientation at $t=1 \mathrm{~s}$ and $t=5 \mathrm{~s}$. Thereupon, the mobile base tries to compensate for that error (bottom plot). This, in turn, leads to a null space motion of the Cartesian impedance task. When releasing the TCP, the remaining platform velocity and the impedance induce a small overshoot before a steady-state is reached again. That effect can be reduced by using a higher stiffness. Another possibility would be to account for the platform velocity within the damping design of the Cartesian impedance (3).

The video which accompanies this paper presents some exemplary scenes showing the performance of the controller. That includes a demonstration of the null space while interacting with a human. Another scene shows the robot reaching for an object which is identified by a camera tracking system.

\section{CONCLUSIONS}

Service and household robotics are expected to increase in significance over the next decades. Therefore, the ability to execute complex tasks is one of the key issues. Additionally, various further aspects have to be tackled simultaneously. That includes the coordination in joint space and the guarantee of safety concerning the robot environment and the own structure. Beside the common approach of solely using planning algorithms, a second field of research focuses on the combination of low-dimensional planning and reactive robot behavior to handle the rest. In this paper, we presented a method of the latter kind for the mobile and multiredundant torque controlled humanoid Justin. Based on a low-dimensional trajectory for the TCP, the robot managed issues like collision avoidance and other subtasks autonomously. Due to the torque control in most of the joints, the robot provided a compliant interaction with its environment and ensured a high level of safety. The approach has been validated through experiments on the real robotic system. Motion generation and reactive behavior were realized in a $1 \mathrm{~ms}$ cycle. As demonstrated in the video, the motions appeared natural. Also the combined behavior of the base and the upper body was shown.
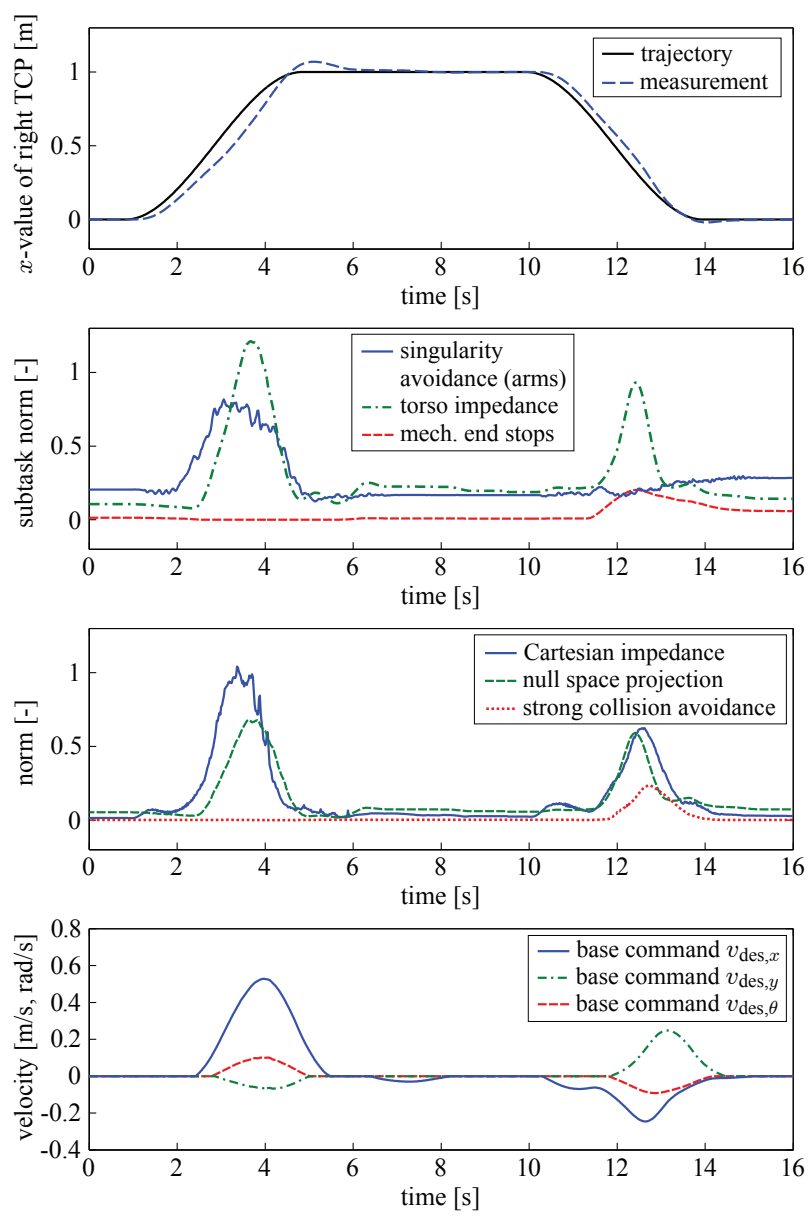

Fig. 6. Robot behavior for a continuous trajectory of the right TCP with an impedance stiffness of $500 \mathrm{~N} / \mathrm{m}$ (translational) and $100 \mathrm{Nm} / \mathrm{rad}$ (rotational) and a damping ratio of 0.7 .

Future work will focus on communication between planner and whole-body motion controller. Insuperable obstacles or local minima due to the reactive robot behavior have to be handled appropriately. Therefore, a feedback from controller to planner is an inevitable feature. A second field of research will address the treatment of various (sub)tasks. The redundancy resolution proposed in this paper is not generic as it accounts for two priority levels on which subtasks have been properly engineered. We are currently working on a dynamic hierarchy-based redundancy resolution to augment and generalize the framework presented in this paper.

\section{REFERENCES}

[1] Y. Sakagami, R. Watanabe, C. Aoyama, S. Matsunaga, N. Higaki, and K. Fujimura, "The intelligent ASIMO: System overview and integration," in Proc. of the 2002 IEEE/RSJ International Conference on Intelligent Robots and Systems, October 2002, pp. 2478-2483.

[2] “PR2," Willow Garage, http://www.willowgarage.com/pr2, July 2011.

[3] T. Asfour, K. Regenstein, J. Schröder, A. Bierbaum, N. Vahrenkamp, and R. Dillmann, "ARMAR-III: An Integrated Humanoid Platform for Sensory-Motor Control," in Proc. of the 6th IEEE-RAS International Conference on Humanoid Robots, December 2006, pp. 169-175.

[4] C. Borst, T. Wimböck, F. Schmidt, M. Fuchs, B. Brunner, F. Zacharias, P. R. Giordano, R. Konietschke, W. Sepp, S. Fuchs, C. Rink, A. AlbuSchäffer, and G. Hirzinger, "Rollin' Justin - Mobile Platform with Variable Base," in Proc. of the 2009 IEEE International Conference on Robotics and Automation, May 2009, pp. 1597-1598. 

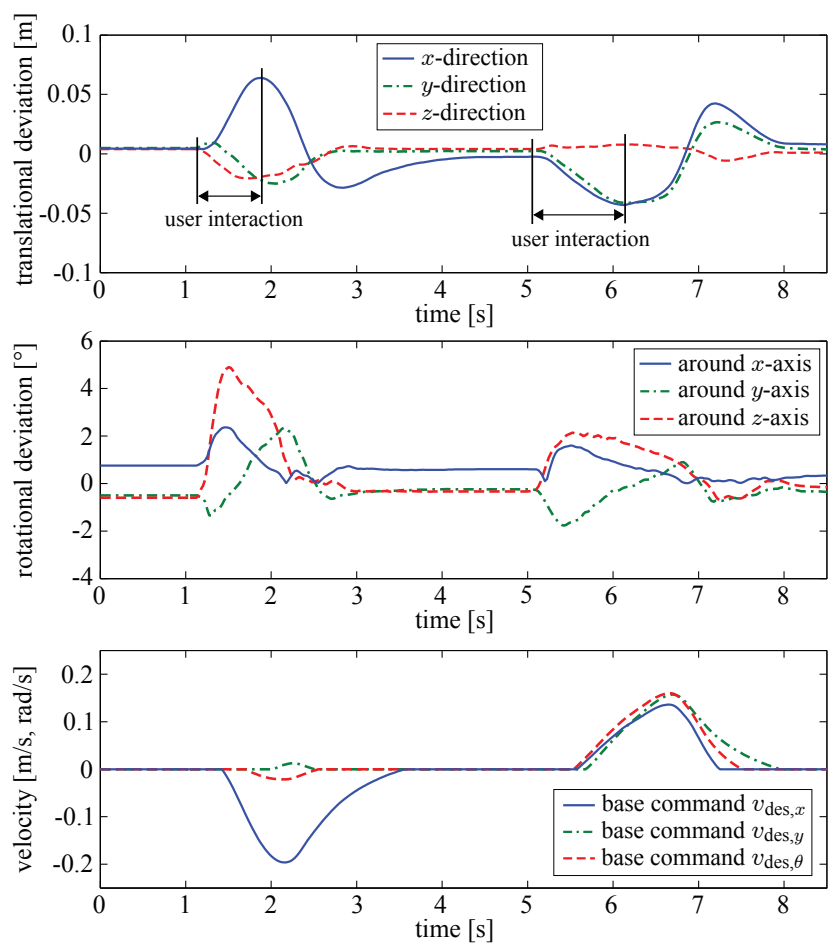

Fig. 7. TCP deviations and platform commands while interacting with a human.

[5] A. Albu-Schäffer, C. Ott, and G. Hirzinger, "A Unified Passivitybased Control Framework for Position, Torque and Impedance Control of Flexible Joint Robots," The International Journal of Robotics Research, vol. 27, no. 1, pp. 23-39, January 2007.

[6] G. B. Hammam, D. E. Orin, and B. Dariush, "Whole-Body Humanoid Control from Upper-Body Task Specifications," in Proc. of the 2010 IEEE International Conference on Robotics and Automation, May 2010, pp. 3398-3405.

[7] A. Jain and C. C. Kemp, "Pulling Open Doors and Drawers: Coordinating an Omni-directional Base and a Compliant Arm with Equilibrium Point Control," in Proc. of the 2010 IEEE International Conference on Robotics and Automation, May 2010, pp. 1807-1814.

[8] K. Nagasaka, Y. Kawanami, S. Shimizu, T. Kito, T. Tsuboi, A. Miyamoto, T. Fukushima, and H. Shimomura, "Whole-body Cooperative Force Control for a Two-Armed and Two-Wheeled Mobile Robot Using Generalized Inverse Dynamics and Idealized Joint Units," in Proc. of the 2010 IEEE International Conference on Robotics and Automation, May 2010, pp. 3377-3383.

[9] M. Behnisch, R. Haschke, and M. Gienger, "Task Space Motion Planning Using Reactive Control," in Proc. of the 2010 IEEE/RSJ International Conference on Intelligent Robots and Systems, October 2010, pp. 5934-5940.

[10] L.-P. Ellekilde and H. I. Christensen, "Control of Mobile Manipulator using the Dynamical Systems Approach," in Proc. of the 2009 IEEE International Conference on Robotics and Automation, May 2009, pp. 1370-1376.

[11] D. H. Shin, B. S. Hamner, S. Singh, and M. Hwangbo, "Motion Planning for a Mobile Manipulator with Imprecise Locomotion," in Proc. of the 2003 IEEE/RSJ International Conference on Intelligent Robots and Systems, October 2003, pp. 847-853.

[12] O. Khatib, "A Unified Approach for Motion and Force Control of Robot Manipulators: The Operational Space Formulation," IEEE Journal of Robotics and Automation, vol. RA-3, no. 1, pp. 43-53, February 1987.
[13] — , "Real-Time Obstacle Avoidance for Manipulators and Mobile Robots," The International Journal of Robotics Research, vol. 5, no. 1, pp. 90-98, Spring 1986.

[14] H. Sugiura, M. Gienger, H. Janssen, and C. Goerick, "Real-Time Collision Avoidance with Whole Body Motion Control for Humanoid Robots," in Proc. of the 2007 IEEE/RSJ International Conference on Intelligent Robots and Systems, October 2007, pp. 2053-2058.

[15] O. Brock and O. Khatib, "Elastic Strips: A Framework for Motion Generation in Human Environments," The International Journal of Robotics Research, vol. 21, no. 12, pp. 1031-1052, December 2002.

[16] N. Mansard, A. Remazeilles, and F. Chaumette, "Continuity of Varying-Feature-Set Control Laws," IEEE Transactions on Automatic Control, vol. 54, no. 11, pp. 2493-2505, November 2009.

[17] N. Mansard, O. Khatib, and A. Kheddar, "A Unified Approach to Integrate Unilateral Constraints in the Stack of Tasks," IEEE Transactions on Robotics, vol. 25, no. 3, pp. 670-685, June 2009.

[18] L. Sentis and O. Khatib, "A Whole-Body Control Framework for Humanoids Operating in Human Environments," in Proc. of the 2006 IEEE International Conference on Robotics and Automation, May 2006, pp. 2641-2648.

[19] C. Ott, O. Eiberger, W. Friedl, B. Bäuml, U. Hillenbrand, C. Borst, A. Albu-Schäffer, B. Brunner, H. Hirschmüller, S. Kielhöfer, R. Konietschke, M. Suppa, T. Wimböck, F. Zacharias, and G. Hirzinger, "A Humanoid Two-Arm System for Dexterous Manipulation," in Proc. of the IEEE/RAS International Conference on Humanoid Robots, December 2006, pp. 276-283.

[20] P. R. Giordano, M. Fuchs, A. Albu-Schäffer, and G. Hirzinger, "On the Kinematic Modeling and Control of a Mobile Platform Equipped with Steering Wheels and Movable Legs," in Proc. of the 2009 IEEE International Conference on Robotics and Automation, May 2009, pp. 4080-4087.

[21] N. Hogan, "Impedance Control: An Approach to Manipulation: Part I - Theory, Part II - Implementation, Part III - Applications," Journal of Dynamic Systems, Measurement, and Control, vol. 107, pp. 1-24, March 1985.

[22] A. Albu-Schäffer, C. Ott, U. Frese, and G. Hirzinger, "Cartesian Impedance Control of Redundant Robots: Recent Results with the DLR-Light-Weight-Arms," in Proc. of the 2003 IEEE International Conference on Robotics and Automation, Sept. 2003, pp. 3704-3709.

[23] C. Ott, A. Albu-Schäffer, A. Kugi, S. Stramigioli, and G. Hirzinger, "A Passivity Based Cartesian Impedance Controller for Flexible Joint Robots - Part I: Torque Feedback and Gravity Compensation," in Proc. of the 2004 IEEE International Conference on Robotics and Automation, April 2004, pp. 2659-2665.

[24] A. Albu-Schäffer, C. Ott, and G. Hirzinger, "A Passivity Based Cartesian Impedance Controller for Flexible Joint Robots - Part II: Full State Feedback, Impedance Design and Experiments," in Proc. of the 2004 IEEE International Conference on Robotics and Automation, April 2004, pp. 2666-2672.

[25] T. Wimböck, C. Ott, and G. Hirzinger, "Impedance Behaviors for Twohanded Manipulation: Design and Experiments," in Proc. of the 2007 IEEE International Conference on Robotics and Automation, April 2007, pp. 4182-4189.

[26] A. Dietrich, T. Wimböck, H. Täubig, A. Albu-Schäffer, and G. Hirzinger, "Extensions to Reactive Self-Collision Avoidance for Torque and Position Controlled Humanoids," in Proc. of the 2011 IEEE International Conference on Robotics and Automation, May 2011, pp. $3455-3462$.

[27] C. Ott, Cartesian Impedance Control of Redundant and FlexibleJoint Robots, ser. Springer Tracts in Advanced Robotics. Springer Publishing Company, Berlin Heidelberg, 2008, vol. 49.

[28] A. Dietrich, T. Wimböck, A. Albu-Schäffer, and G. Hirzinger, "Singularity Avoidance for Nonholonomic, Omnidirectional Wheeled Mobile Platforms with Variable Footprint," in Proc. of the 2011 IEEE International Conference on Robotics and Automation, May 2011, pp. 6136-6142. 